09

\title{
Генерация аттосекундного импульса на основе коллективного спонтанного излучения слоя трехуровневых атомов, возбуждаемых парой униполярных импульсов
}

\author{
(C) Р.М. Архипов ${ }^{1,2,3}$, М.В. Архипов ${ }^{1,2}$, И. Бабушкин ${ }^{4,5}$, А.В. Пахомов ${ }^{1,2}$, Н.Н. Розанов ${ }^{2,3}$ \\ ${ }^{1}$ Санкт-Петербургский государственный университет, \\ 199034 Санкт-Петербург, Россия \\ ${ }^{2}$ Университет ИТМО, \\ 197101 Санкт-Петербург, Россия \\ ${ }^{3}$ ФТИ им. А.Ф. Иофрфе, \\ 194021 Санкт-Петербург, Россия \\ ${ }^{4}$ Institute of Quantum Optics, Leibniz University Hannover, \\ 30167 Hannover, Germany \\ ${ }^{5}$ Cluster of Excellence PhoenixD (Photonics, Optics, and Engineering - Innovation across Disciplines), \\ 30167 Hannover, Germany \\ e-mail: arkhipovrostislav@gmail.com
}

Поступила в редакцию 22.06.2020 г.

В окончательной редакции 22.06.2020 г.

Принято к публикации 06.07.2020 г.

\begin{abstract}
Недавно для генерации предельно коротких импульсов был предложен метод когерентного контроля поляризации среды, основанный на возбуждении колебаний атомарной поляризации и их последующей остановки с помощью пары ультракоротких импульсов. Так называемый остановленный импульс поляризации среды, возникающий в промежутке между ее возбуждением и девозбуждением, может являться источником предельно короткого импульса излучения. В данной работе теоретически рассматривается указанная возможность генерации изолированного аттосекундного ультрафиолетового импульса в трехуровневой резонансной среде, параметры которой соответствуют атому водорода, возбуждаемого парой униполярных рентгеновских импульсов. При этом механизм генерации является „антенным“, то есть обусловлен коллективным спонтанным излучением предварительно сфазированных атомов в отсутствие заметного затухания их свободной поляризации.
\end{abstract}

Ключевые слова: коллективное спонтанное излучение, когерентный контроль атомарной поляризации, аттосекундные импульсы, униполярные импульсы, рентгеновские импульсы, атом водорода.

DOI: $10.21883 /$ OS.2020.11.50176.182-20

\section{Введение}

Получение предельно коротких импульсов аттосекундной длительности $\left(1 \mathrm{as}=10^{-18} \mathrm{~s}\right)$ за последние годы стало одной из центральных тем современной физики и оптики [1-12]. Периоды движений электронов в атомах, молекулах и твердых телах могут лежать в аттосекундном диапазоне, и поэтому эти импульсы используются для мониторинга движения электронов и управления им $[1,3,6,10]$. В последнее время возрос интерес к применению аттосекундных импульсов для изучения динамики электронов в твердотельных наночастицах [11], в электронной микроскопии [12] и управления динамики когерентности связанных электронов в атомах [13]. Экспериментально аттосекундные импульсы получаются с помощью генерации гармоник высокого порядка (ГГВП) (high order harmonics generation, HHG), которая возникает при взаимодействии инфракрасных фемтосекундных лазерных импульсов с различными веществами [1-5]. Физические процессы, возникающие при ГГВП, описываются в литературе на основе „трех- ступенчатой модели“ („three-step model“) [1]. Именно, происходит ионизация электрона в лазерном поле, его ускорение в поле импульса и последующая рекомбинация с родительским ионом. Когерентное сложение возникающих при этом нечетных гармоник центральной частоты возбуждающего импульса приводит к генерации последовательности аттосекундных импульсов с частотой следования, равной несущей частоте возбуждающего импульса [1-5]. Также разработаны различные модификации метода ГГВП, такие как метод стробирования (gating technique) и др., которые позволяют получить изолированный аттосекундный импульс $[1,3,5]$.

Обычно методы получения аттосекундного импульса, основанные на ГГВП, требуют громоздких установок [1-12]. Поэтому в настоящее время актуальным является поиск альтернативных методов получения аттосекундных импульсов. Недавно были получены аттосекундные импульсы в оптическом диапазоне с помощью Фурье-синтеза частот накачки [6] и предложены способы получения таких импульсов за счет ускорения 
электронов, выбиваемых из тонкой мишени с помощью фемтосекундного лазерного импульса $[4,8]$.

Следует отметить и предложенный в работе [14] способ генерации субциклового аттосекундного импульса при отражении одноциклового импульса от тонкой пленки металла или диэлектрика в одномерной геометрии. Параллельно был разработан метод генерации предельно коротких импульсов за счет возбуждения и последующего девозбуждения (остановки) поляризации тонкого слоя среды (метод когерентного контроля поляризации, МККП) парой высокочастотных импульсов, частота которых выше рассматриваемого резонансного перехода среды [15-18]. Длительность импульсов возбуждения и интервал между ними короче времен релаксации инверсии населенностей уровней и поляризации в среде $\left(T_{1}\right.$ и $\left.T_{2}\right)$ и поэтому когерентность среды не успевает разрушиться за время действия импульсов. Идея МККП заключается в том, что первый импульс возбуждает собственные (свободные) осцилляции поляризации среды. После первого импульса происходит медленное затухание свободной поляризации среды [19-22]. Затем второй импульс приводит к остановке эти колебания поляризации при условии, что он приходит с задержкой, кратной нечетному числу полупериодов колебаний поляризации среды. В промежутке между высокочастотными импульсами возникает импульс так называемой остановленной поляризации среды, создающей электромагнитное излучение [19-22]. В принципе, если говорить не об аттосекундных, а об уже освоенных нано- и субнаносекундных длительностях импульсов, то этот метод, аналогичный используемому в фазированных антенных решетках (пассивных и/или активных; см., например, [23-25]) и известен в оптическом и радиодиапазонах для различных опто- и радиоэлектронных импульсных систем [26].

Реализующийся процесс излучения является коллективным - все атомы вносят одинаковый вклад в поляризацию среды, и поля излучения от всех диполей складываются в фазе, если размер системы много меньше длины излучаемой волны. При большой созданной населенности возбужденных уровней среды, особенно при наличии инверсии их населенностей, импульс поляризации с достаточной большой протяженностью во времени и/или пространстве может приводить к нелинейным явлениям сверхизлучательного типа, которые связанны с коллективным спонтанным излучением анасамбля атомов и впервые были указаны Дике [27]. Впоследствии такого рода нелинейные явления были изучены различными авторами в оптически толстых образцах для различных материалов и разных ансамблей излучателей, в частности, радиоизлучающих спинов электронов или ядер [28-30] и оптических наноизлучателей, в том числе нанолазеров [31-32] (о сверхизлучении см. подробнее в [26,33-40] и цитированной там литературе).

В данной работе мы не будем рассматривать нелинейные явления и ограничимся линейной задачей о так называемом „антенном“ (или коллективном) механизме излучения сильно ограниченного в пространстве и во времени импульса поляризации среды, созданного по схеме МККП. Предложенная реализация антенного механизма излучения может иметь самые разнообразные применения, скажем, для генерации униполярных импульсов [15-17], например, в ТГц дипазоне частот [17], и одноцикловых ТГц импульсов [18]. В работе [41] была рассмотрена возможность получения с помощью подобного метода управления поляризацией среды УФ аттосекундного импульса с использованием атомов гелия, возбуждаемых рентгеновскими униполярными импульсами. Проведенный там анализ был ограничен двухуровневым приближением на том основании, что расстояние между соседними уровнями быстро уменьшается с ростом главного квантового числа. Поэтому все низкочастотные переходы, кроме основного наиболее высокочастотного, слабо возбуждаются и их вклад в отклик ансамбля атомов гелия сводится к добавлению поправок малой амплитуды, которые можно не учитывать. Однако, для более детального анализа поляризационного отклика и генерируемого им ультрафиолетового излучения необходим учет большего числа уровней среды.

В настоящей работе исследована возможность получения одиночного аттосекундного импульса в результате излучения остановленной поляризации, созданной с помощью МККП в трехуровневой оптически тонкой среде, параметры которой соответствуют переходам в атоме водорода (в пренебрежении несущественным для данной задачи неоднородным, например доплеровским, уширением всех возбужденных переходов). Рассматриваемое явление связано с кратковременным коллективным излучением предварительно сфазированных атомов. Установлено, что в условиях низкой и практически неизменной населенности возбужденных состояний атомов мощность их коллективного излучения пропорциональна квадрату плотности излучающих атомов, в полной аналогии с известным антенным механизмом излучения радиосистем и электронных спинов [42-46].

В качестве импульсов возбуждения, как и в [41], предполагается использовать сверхкороткие квазиуниполярные рентгеновские импульсы. Рентгеновские импульсы в аттосекундном дипазоне длительностей получаются на сегодняшний день, например, в лазерах на своболных электронах; см. обзор [47] и приведенную там литературу. Длительность рентгеновских импульсов в лазере на свободных электронах в режиме синхронизации мод может быть даже в зептосекундном диапазоне $\left(1 \mathrm{zs}=10^{-21} \mathrm{~s}\right)$ [48]. Если такие импульсы излучаются при ускорении свободных электронов, то они, в принципе, могут иметь постоянную составляющую поля, то есть являться униполярными [49-51]. Генерации униполярных импульсов и их применению в оптике посвящены различные обзоры $[16,49-51]$. В настоящее время уже не вызывает сомнения, что униполярные импульсы способны оказывать более эффективное воздействие на квантовые объекты, чем одноцикловые и 
многоцикловыми биполярные импульсы [50-54]. Поэтому униполярные импульсы могут найти широкое применение в различных областях физики, например, для эффективного управления состоянием квантовых объектов [52-54], ускорения заряженных частиц [55], селективного возбуждения квантовых состояний атомных систем [56-57], а также в голографии [58]. В данной же работе мы фокусируемся на еще одном возможном применении униполярных импульсов - формировании аттосекундного импульса коллективного спонтанного излучения в трехуровневой среде.

\section{Получение аттосекундного импульса в тонком слое трехуровневой резонансной среды}

Идея изучаемого метода аналогична используемой для получения аттосекундного импульса в двухуровневой среде [41], она иллюстрируется на рис. 1. Тонкий слой атомов водорода, который моделируется в трехуровневом приближении (схема уровней приведена на рисунке), располагается перпендикулярно оси $z$, между точками $z_{1}$ и $z_{2}$. Он возбуждается парой униполярных импульсов в рентгеновском диапазоне. Эти импульсы, как и генерируемое ультрафиолетовое излучение, рассматриваются в одномерном приближении - плоские волны, распространяющиеся вдоль оси $z$. Интервал следования рентгеновских импульсов равен половине периода $T_{0} / 2$ перехода из основного состояния с квантовым числом $n=1$ в первое возбужденное с $n=2$ ультрафиолетовой серии Лаймана водорода с длиной волны $121.6 \mathrm{~nm}$.

В случае атомов гелия в [41] было использовано двухуровневое приближение, так как высоко возбужденные уровни лежат относительно близко к первому возбужденному, и расстояние между уровнями быстро уменьшается с ростом главного квантового числа $n$. Поэтому их вклад в колебания поляризации среды будет заключаться лишь в наличии хвоста малой амплитуды, который можно не учитывать. Аналогична структура уровней у атома водорода. Ниже для полноты описания будем учитывать большее число уровней и рассмотрим трехуровневую схему, см. рис. $1, b$, параметры которой соответствуют атому водорода. Также, как показали результаты численного интегрирования временного уравнения Шредингера, приведенные в работе [41], вероятность ионизации атома в таких ультракоротких импульсах очень мала (порядка $10^{-11}$ ), несмотря на то что энергия фотонов больше потенциала ионизации. Это позволяет не учитывать ионизацию в нашем рассмотрении и делает оправданным применение малоуровневого приближения.

Взаимодействие трехуровневой среды с парой субцикловых импульсов подчиняется следующей системе
Incident unipolar

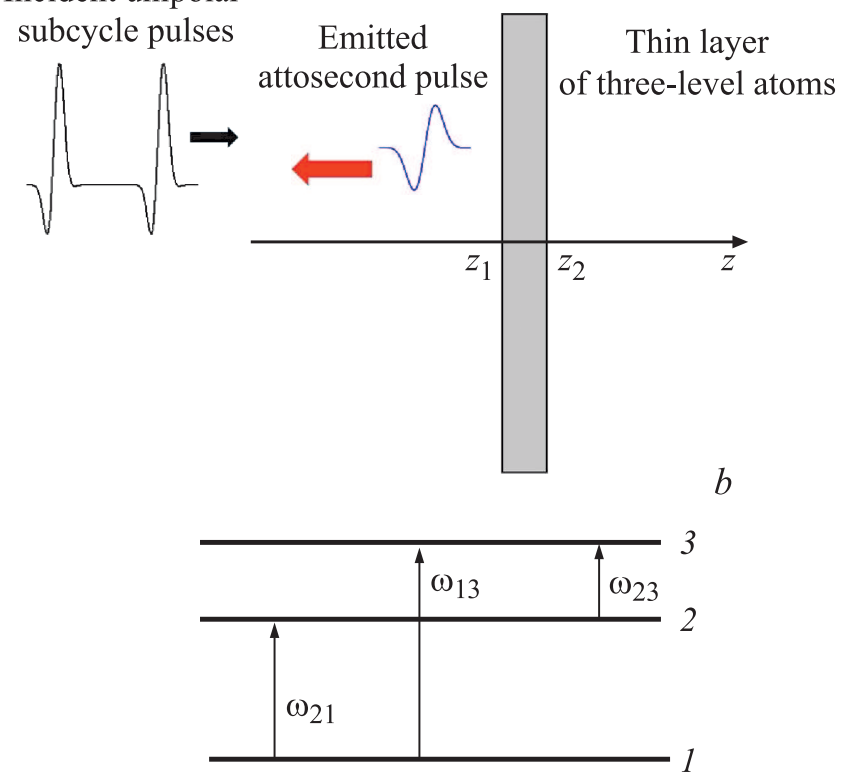

Рис. 1. Схема получения аттосекундного импульса в трехуровневой среде, возбуждаемой парой субцикловых импульсов $(a)$. Схема рабочих уровней среды $(b)$.

уравнений для элементов матрицы плотности [59,60]:

$$
\begin{aligned}
\frac{\partial}{\partial t} \rho_{21}= & -i \omega_{21} \rho_{21}-i \frac{d_{12}}{\hbar} E\left(\rho_{22}-\rho_{11}\right)-i \frac{d_{13}}{\hbar} E \rho_{23} \\
& +i \frac{d_{23}}{\hbar} E \rho_{31}, \\
\frac{\partial}{\partial t} \rho_{32}= & -i \omega_{23} \rho_{32}-i \frac{d_{23}}{\hbar} E\left(\rho_{33}-\rho_{22}\right)-i \frac{d_{12}}{\hbar} E \rho_{31} \\
& +i \frac{d_{13}}{\hbar} E \rho_{21}, \\
\frac{\partial}{\partial t} \rho_{31}= & -i \omega_{13} \rho_{31}-i \frac{d_{13}}{\hbar} E\left(\rho_{33}-\rho_{11}\right)-i \frac{d_{12}}{\hbar} E \rho_{32} \\
& +i \frac{d_{23}}{\hbar} E \rho_{21}, \\
\frac{\partial}{\partial t} \rho_{11}= & i \frac{d_{12}}{\hbar} E\left(\rho_{21}-\rho_{21}^{*}\right)-i \frac{d_{13}}{\hbar} E\left(\rho_{13}-\rho_{13}^{*}\right), \\
\frac{\partial}{\partial t} \rho_{22}= & -i \frac{d_{12}}{\hbar} E\left(\rho_{21}-\rho_{21}^{*}\right)-i \frac{d_{23}}{\hbar} E\left(\rho_{23}-\rho_{23}^{*}\right), \\
\frac{\partial}{\partial t} \rho_{33}= & i \frac{d_{13}}{\hbar} E\left(\rho_{13}-\rho_{13}^{*}\right)+i \frac{d_{23}}{\hbar} E\left(\rho_{23}-\rho_{23}^{*}\right) .
\end{aligned}
$$

Уравнения (1)-(3) описывают эволюцию недиагональных элементов матрицы плотности $\rho_{21}, \rho_{32}, \rho_{31}$, которые связаны с поляризацией среды. Уравнения (4)-(6) описывают эволюцию диагональных элементов матрицы плотности $\rho_{11}, \rho_{22}, \rho_{33}$, имеющих смысл заселенностей 1-го, 2-го и 3-го состояний среды соответственно. Параметры $d_{12}, d_{13}, d_{23}$ - дипольные моменты соответствующих переходов атомов среды, $\omega_{21}, \omega_{23}, \omega_{13}-$ 
частоты переходов, $\hbar$ - приведенная постоянная Планка, $E$ - напряженность электрического поля излучения (линейно поляризованного). Ввиду малой длительности импульсов мы пренебрегли релаксационными членами и для простоты исключили возможное неоднородное уширение всех трех дипольно разрешенных спектральных линий.

Пусть среда возбуждается парой квазиуниполярных рентгеновских импульсов:

$$
\begin{aligned}
E_{x}(t)= & E_{0 X} e^{\frac{-t^{2}}{\tau^{2}}} \cos \left(\omega_{x} t+\phi\right)+E_{0 X} e^{\frac{-\left(t-T_{d}\right)^{2}}{\tau^{2}}} \\
& \times \cos \left(\omega_{x}\left[t-T_{d}\right]+\phi\right) .
\end{aligned}
$$

Здесь $\omega_{x}$ - несущая частота рентгеновских импульсов, $\tau$ - их эффективная длительность, $\phi-$ фаза (carrier envelope phase, CEP). Задержка между импульсами $T_{d}$ в нашей задаче равна половине периода перехода атома водорода из основного состояния в первое возбужденное (главная линия серии Лаймана) $T_{0} / 2\left(T_{0}=405\right.$ as). Поляризация одиночного атома среды с координатой $z$ связана с недиагональными элементами матрицы плотности соотношением

$$
p(z, t)=2 d_{12} \Re \rho_{12}+2 d_{13} \Re \rho_{13}+2 d_{23} \Re \rho_{23}
$$

и подбором амплитуды, длительности и фазы второго девозбуждающего импульса (7) может быть сделанной очень малой после его прохождения через данный атом. Впрочем, последнее, как будет ясно из дальнейшего, в достаточной мере выполняется даже при указанном возбуждении двумя одинаковыми рентгеновскими импульсами (7).

Эволюция электрического поля описывается одномерным волновым уравнением

$$
\frac{\partial^{2} E(z, t)}{\partial z^{2}}-\frac{1}{c^{2}} \frac{\partial^{2} E(z, t)}{\partial t^{2}}=\frac{4 \pi}{c^{2}} \frac{\partial^{2} P(z, t)}{\partial t^{2}},
$$

в котором $E-$ напряженность электрического поля с фиксированной линейной поляризацией, $P=N_{0} p-$ поляризация ансамбля атомов с концентрацией $N_{0}, c-$ скорость света в вакууме. Мы также рассматриваем одномерную задачу, поскольку фактически речь идет не о распространении излучения, а об антенном механизме излучения ансамбля диполей с предварительно определенной атомарной поляризацией, не вносящей заметные отличия диэлектрической проницаемости от единицы (обычно такое приближение справедливо для широких пучков на расстояниях, меньших дифракционной длины). В рассматриваемом приближении и геометрии рис. 1 аналитическое решение волнового уравнения (9) для поля аттосекундного импульса, генерируемого заданной кратковременной поляризацией $P(z, t)$, имеет вид [14]:

$$
E_{a}(z, t)=-\frac{2 \pi}{c} \int_{z_{1}}^{z_{2}} \frac{\partial}{\partial t} P\left(z^{\prime}, t-\frac{\left|z-z^{\prime}\right|}{c}\right) d z^{\prime} .
$$
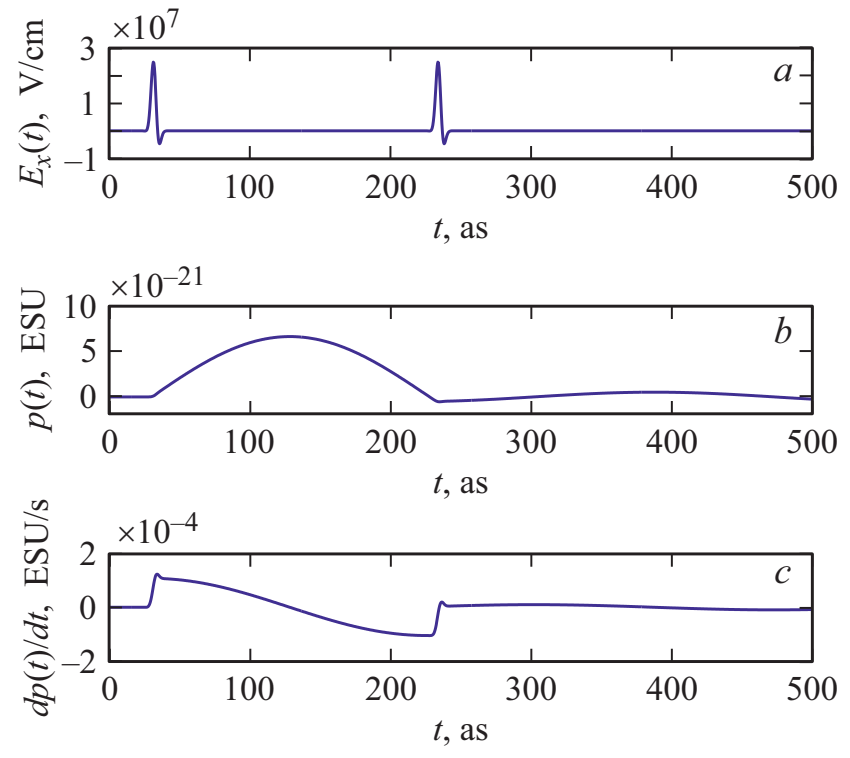

Рис. 2. Полуцикловые рентгеновские импульсы возбуждения $E_{x}$, следующие с интервалом $T_{0} / 2 \quad\left(T_{0}=405\right.$ as $)(a)$; поляризация единичного атома $p(b)$, ее производная $d p / d t(c)$. Параметры импульсов возбуждения и среды приведены в таблице.

Как известно, для слоя конечной толщины в пренебрежении эффектами запаздывания это решение можно записать в виде разложения по модам Лиенарда-Вихерта (см. [61-63]). Если среда представляет собой оптически тонкий слой атомов, толщиной $L \ll \lambda_{12,23,13}$, решение (10) упрощается и имеет „локальный“ вид [41]

$$
E_{a}(t)=-\frac{2 \pi}{c} L N_{0} \frac{\partial}{\partial t} p(t) .
$$

Ниже мы будем рассматривать именно тонкий слой толщиной $L$ меньше минимальной длины волны переходов $12,23,13$, а именно $L \approx(1 / 4) \lambda_{12}$.

Рис. 2 показывает импульсы возбуждения $(a) E_{x}(t)$, поляризацию одиночного атома $(b) p(t)$ и ее производную по времени $(c) \frac{\partial}{\partial t} p(t)$, форма которой совпадает с аттосекундным импульсом ультрафиолетового излучения. Этот рисунок получен в результате численного решения системы уравнений для матрицы плотности трехуровневой среды (1)-(6) с полем, рентгеновских импульсов (7) при параметрах среды и поля, указанных в таблице. Частоты переходов и дипольные моменты трехуровневой среды брались согласно [64,65] такими же, как в атоме водорода.

Поляризация возбуждается парой одинаковых импульсов, следующих с интервалом $T_{0} / 2$. Она приближенно имеет вид полуволны, рис. 2, $b$, поскольку первый импульс возбуждает колебания атомной поляризации, а второй импульс девозбуждает (останавливает) их практически полностью на основном переходе 12 и в значительной мере на двух других переходах 23 и 13. Имеющийся на рисунке осциллирующий хвост объясняется в 
Параметры модели, используемые в расчетах $\left(1 \mathrm{D} \approx 3.335 \cdot 10^{-30} \mathrm{~K} \cdot \mathrm{m}\right)$

\begin{tabular}{c|c}
\hline $\begin{array}{c}\text { Амплитуда рентгеновских импульсов } \\
\text { возбуждения }\end{array}$ & $E_{0 X}=3 \cdot 10^{7} \mathrm{~V} / \mathrm{cm}$ \\
\hline Частота (длина волны) & $\omega_{x}=3.77 \cdot 10^{17} \mathrm{rad} / \mathrm{s}\left(\right.$ длина волны $\left.\lambda_{X}=5 \mathrm{~nm}\right)$ \\
\hline Длительность импульсов возбуждения & $\phi=3.2 \mathrm{as}$ \\
\hline Фаза импульсов возбуждения (СЕР) & $\omega_{21}=1.55 \cdot 10^{16} \mathrm{rad} / \mathrm{s}\left(\lambda_{21}=121.6 \mathrm{~nm}\right)$ \\
\hline Частота перехода 12 в атоме водорода (длина \\
волны перехода) & $d_{12}=3.27 \mathrm{D}$ \\
\hline Дипольный момент перехода 12 & $\omega_{13}=1.837 \cdot 10^{16} \mathrm{rad} / \mathrm{s}\left(\lambda_{13}=102.6 \mathrm{~nm}\right)$ \\
\hline Частота перехода 13 в атоме водорода (длина & $d_{13}=1.31 \mathrm{D}$ \\
\hline волны перехода) & $\omega_{23}=2.87 \cdot 10^{15} \mathrm{rad} / \mathrm{s}\left(\lambda_{23}=656.6 \mathrm{~nm}\right)$ \\
\hline Чипольный момент перехода 13 & $d_{23}=7.78 \mathrm{D}$ \\
\hline вистота перехода 23 в атоме водорода (длина & $N_{0}=10^{19} \mathrm{~cm}{ }^{-3}$ \\
\hline Концентрация частиц в слое & $L=30 \mathrm{~nm}$
\end{tabular}

основном вкладом резонансного перехода 13 с периодом $T_{13}=342$ as, на $17 \%$ отличающимся от $T_{0}=405$ as, и в 2.5 раза меньшим дипольным моментом. В целом после прохождения второго импульса возбуждения атомарная поляризация падает более чем на порядок величины и интенсивность генерируемого ей излучения составляет лишь доли процента от интенсивности излучения в интервале $T_{0} / 2$ между импульсами возбуждения. Поэтому учет третьего уровня несильно влияет на управляемую поляризацию.

Для уяснения сказанного на рис. 3 приведены вклады в полную поляризацию от каждого из рассматриваемых переходов $2 d_{12} \Re \rho_{12}, 2 d_{13} \Re \rho_{13}, 2 d_{23} \Re \rho_{23}$ (рис. $\left.3, a, b, c\right)$ и их сумма - полная поляризация $p(t)-($ рис. $3, d)$.

Согласно этому рисунку, наибольший вклад в поляризации дает резонансный переход 1-2 (рис. 3,a). Амплитуда вклада от перехода $1-3$ примерно в 6 раз меньше (рис. $3, b)$. Вклад от перехода 2-3 имеет амплитуду на 2 порядка меньше амплитуды вклада перехода 1-2 (рис. 3,c), и он полностью исчезает после выключения импульсов. Поэтому наибольший вклад в поляризацию среды дает резонансный переход 1-2.

Форму полуволны поляризации между импульсами в случае двухуровневой среды с частотой перехода $\omega_{0}$ можно приближенно описать синусоидой $[15,41]$ :

$$
p(t)=A \sin \omega_{0} t, A \equiv \frac{2 d_{12}^{2}}{\hbar} \int_{-\infty}^{\infty} E_{x}(t) \cos \omega_{0} t d t
$$

В отсутствие второго импульса данная поляризация существует конечное время $T_{2}$, пока когерентность среды не разрушится (явление затухания свободной поляризации, free induction decay [19-22]). Импульс поляризации, существующий между рентгеновскими импульсами возбуждения является источником импульса генерируемого поля. Входящая в (11) производная поляризации в случае двухуровневой среды может быть описана одним полуциклом колебаний косинусоиды:

$$
\dot{p}=\omega_{0} A \cos \omega_{0} t
$$

Аналогичная косинусоида получается и в трехуровневой среде, см. рис. 2,c, поскольку вклад дополнительных переходов незначителен. Форма производной поляризации на рис. 2,c, согласно формуле (11), должна повторять форму аттосекундного импульса. Поэтому генерируемый импульс, как и в двухуровневой среде, имеет вид одного полуцикла колебаний и длительность $T_{0} / 2 \sim 200$ as. При данных амплитудах рентгеновских импульсов возбуждения уровни 2 и 3 среды заселяются очень мало, так как для всех резонансных переходов 12, 23 и 13 среды длительность импульсов меньше периодов осцилляций Раби, величины которых порядка 20, 100 и $50 \mathrm{fs}$ для рассматриваемых переходов соответственно. Поэтому в среде не только нет инверсии населенностей и, следовательно, сверхизлучения Дике, но и пренебрежимо мало падение населенностей возбужденных уровней за время $T_{0} / 2$ высвечиваемого импульса, а следовательно, отсутствуют нелинейные эффекты.

Используя (11)-(13) и усредняя за период колебания квадрат выражения (11), получим полную энергию излученного аттосекундного импульса (в обе стороны оси $z$ 

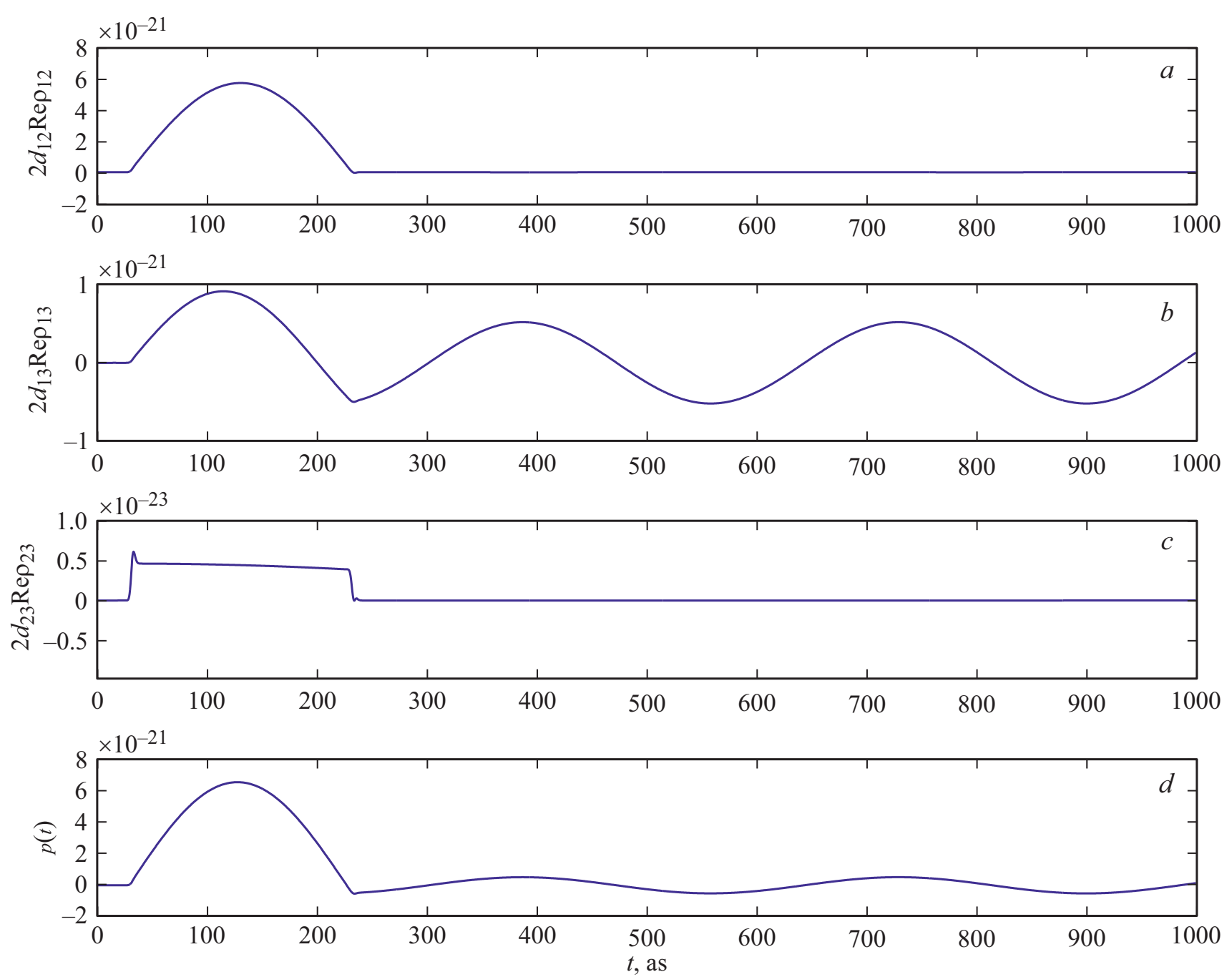

Рис. 3. Временная зависимость вкладов в поляризацию $(a): 2 d_{12} \Re \rho_{12},(b): 2 d_{13} \Re \rho_{13},(c): 2 d_{23} \Re \rho_{23}$ и $(d)$ : их сумма - полная поляризация $p(t)$.

в расчете на единичную площадку поперек нее):

$$
W=\frac{\pi^{3} L^{2} N_{0}^{2} A^{2}}{2 \lambda_{0}} .
$$

Видно, что энергия аттосекундного импульса пропорциональна квадрату концентрации излучающих атомов, что говорит об антенном (коллективном) механизме рассматриваемого излучения. Подчеркнем, что в нашем случае излучение аттосекундного импульса, связанное с затуханием свободной поляризации среды (т.е. фактически с кратковременным коллективное спонтанным излучением), происходит между возбуждающими импульсами в отсутствие возбуждения атомов водорода в слое с толщиной меньше длины волны излучения.

Рис. 4 иллюстрирует рассчитанные по формуле (11) формы генерируемых УФ аттосекундных импульсов при различных значениях фазы рентгеновских импульсов возбуждения СЕР $\phi$.
Согласно этому рисунку, амплитуда генерируемого импульса не сильно зависит от СЕР импульсов возбуждения. Расчеты показывают, что энергетическая эффективность генерации $\frac{\int E_{a}^{2}(t) d t}{\int E_{x}^{2}(t) d t}$ при данных параметрах задачи составляет $10^{-9}$, длительность импульсов генерации порядка 200 as, максимальная амплитуда порядка $100 \mathrm{~V} / \mathrm{cm}$. Для повышения эффективности следует использовать более плотные и/или толстые слои. Однако, как показывают предварительные расчеты, динамика системы в толстом слое существенно иная, чем в случае тонкого слоя. Поэтому рассмотрение поведения системы в оптически толстом слое выходит за рамки настоящей работы, а анализ возможности получения эффективности генерации выше достигнутой с помощью ГГВП $\left(10^{-4}-10^{-6}\right)$ требует дальнейших исследований.

Рис. 5 иллюстрирует численно расчитанную зависимость энергетической эффективности генерации $\frac{\int E_{a}^{2}(t) d t}{\int E_{x}^{2}(t) d t}$ 


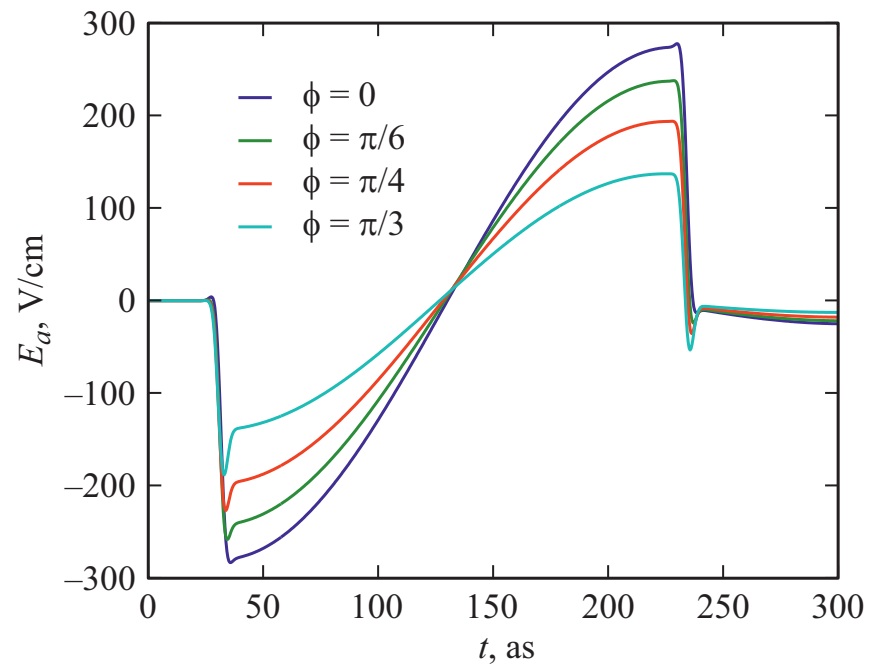

Рис. 4. Рассчитанные формы аттосекундных импульсов излучения $E_{a}$ при различных значениях СЕР накачки $\phi$. Параметры указаны в таблице.

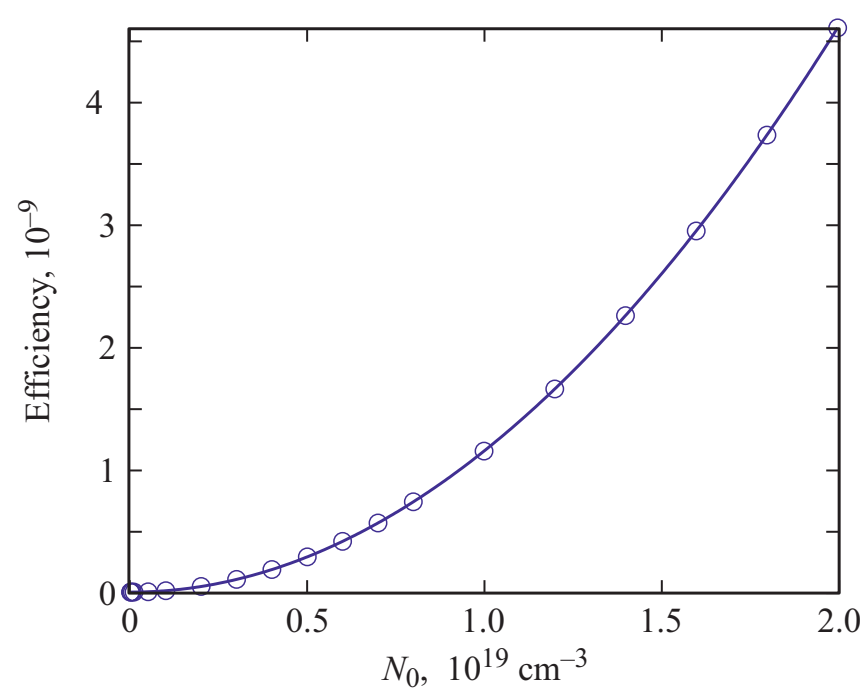

Рис. 5. Зависимость энергетической эффективности генерации импульсов от концентрации атомов в слое $N_{0}$. Остальные параметры расчета указаны в таблице.

от плотности атомов в слое $N_{0}$. Видно, что энергия генерируемого импульса квадратично зависит от концентрации, что вновь говорит о коллективной природе рассматриваемого излучения.

\section{Заключение}

В данной работе была рассмотрена возможность получения УФ аттосекундного импульса в трехуровневой оптически тонкой среде, имеющей параметры, соответствующие переходам в атоме водорода. Источником излучения является свободно эволюционирующий импульс поляризации среды, возникающий при возбуждении и последующем девозбуждении (остановке) УФ колебаний атомных диполей с помощью пары униполярных рентгеновских импульсов. Излучение аттосекундного импульса происходит коллективно при участии всех атомов среды в оптически тонком образце и в отсутствие возбуждающего поля. Интенсивность получаемого ультрафиолетового импульса пропорциональна квадрату концентрации частиц в слое и соответствует антенному (коллективному) механизму излучения в условиях кратковременного существования дипольных осцилляций атомных излучателей с длительностью порядка полупериода основного перехода в атоме водорода.

Рассмотренный в данной работе метод получения одиночного УФ аттосекундного импульса за счет коллективного спонтанного излучения атомов среды, сфазированно возбужденных парой униполярных импульсов, может служить альтернативным методом по отношению к генерации гармоник высокого порядка (ГГВП), что тем самым расширяет возможности применения униполярного света в физике.

\section{Благодарность}

Авторы выражают благодарность Вл.В. Кочаровскому, Е.Р. Кочаровской и С.А. Пулькину за полезные обсуждения и ценные замечания.

\section{Финансирование работы}

Исследования выполнены при финансовой поддержке РНФ в рамках научного проекта № 17-19-01097-П.

\section{Конфоликт интересов}

Авторы заявляют, что у них нет конфликта интересов.

\section{Список литературы}

[1] Corkum P.B., Krausz F. // Nature Physics. 2007. V. 3 (6). P. 381.

[2] Krausz F., Ivanov M. // Rev. Mod. Phys., 2009. V. 81. P. 163.

[3] Nisoli M. , Sansone G. // Progress in Quantum Electronics 2009. V. 33. P. $17-59$

[4] Wu H.-C., Meyer-ter-Vehn J. // Nature Photon. 2012. V. 6. P. 304.

[5] Calegari F. et al. // Journal of Physics B: Atomic, Molecular and Optical Physics. 2016. V. 49. N 6. P. 062001.

[6] Hassan M.T., Luu T.T., Moulet A., Raskazovskaya O. et al. // Nature. 2016. V. 530. P. 66.

[7] Gaumnitz T., Jain A., Pertot Y., Huppert M., Jordan I., Ardana-Lamas F., Wörner H.J. // Optics Express. 2017. V. 25. N 22. P. 27506-27518.

[8] Xu J., Shen B., Zhang X. et al. // Sci. Rep. 2018. V. 8. P. 2669.

[9] Tibai Z., Tóth G., Nagyvóradi A., Gyöngy A., Fülöp J.A., Hebling J., Almási G. Frontiers in Physics. 2018. V. 6. P. 140.

[10] Ramasesha K., Leone S.R., Neumark D.M. // Annu. Rev. Phys. Chem. 2016. V. 67. P. 41. 
[11] Krüger M., Lemell C., Wachter G., Burgdörfer J., Hommelhoff P. // J. Phys. B: Atomic, Molecular and Optical Physics. 2018. V. 51. N 17. P. 172001.

[12] Garg M., Kern K. // Science. 2020. V. 367. N 6476. P. $411-415$.

[13] Jiang W.C., Tong X.M., Pazourek R., Nagele S., Burgdörfer J. // Physical Review A. 2020. V. 101. N 5. P. 053435.

[14] Arkhipov M.V., Arkhipov R.M., Pakhomov A.V., Babushkin I.V., Demircan, A., Morgner, U., Rosanov N.N. // Opt. Lett. 2017. V. 42. N 11. P. 2189-2192.

[15] Pakhomov A.V., Arkhipov R.M., Babushkin I.V., Arkhipov M.V., Tolmachev Yu.A., Rosanov N.N. // Phys. Rev. A. 2017. V. 95. P. 013804

[16] Архипов Р.М., Пахомов А.В., Архипов М.В., Бабушкин И., Толмачев Ю.А., Розанов Н.Н. // Письма в ЖЭТФ. 2017. T. 105. № 6. C. 388-400; Arkhipov R.M., Pakhomov A.V., Babushkin I., Tolmachev Yu.A., Rosanov N.N. // JETP Lett. 2017. V. 105. № 6. P. 408-418.

[17] Pakhomov A.V., Arkhipov R.M., Arkhipov M.V., Demircan A., Morgner U., Rosanov N.N. // Sci. Rep. 2019. V. 9 (1). P. 7444.

[18] Arkhipov R.M., Pakhomov A.V., Arkhipov M.V., Demircan A., Morgner U., Rosanov N.N., Babushkin I. // Phys. Rev. A. 2020. V. 101. N 4. P. 043838.

[19] Hahn E.L. // Phys. Rev. 1950. V. 77. P. 297.

[20] Brewer R.G., Shoemaker R.L. // Phys. Rev. Lett. 1971. V. 27. P. 631 .

[21] Brewer R.G., Shoemaker R.L. // Phys. Rev. A. 1972. V. 6. P. 2001.

[22] Аллен Л., Эберли Дж. Оптический резонанс и двухуровневые атомы. М.: Мир, 1978; Allen L., Eberly J.H. Optical resonance and two-level atoms. N. Y.: Wiley, 1975.

[23] Воскресенский Д.И. и др. Антенны и устройства СВЧ. 2-е изд. М.: Радиотехника, 2006.;

[24] Fujimoto K. Mobile antenna systems handbook. Third edition. 2008 Artech. House, Inc., 2008.

[25] Шишлов А.В. и др. ЖУРНАЛ РАДИОЭЛЕКТРОНИКИ, ISSN 1684-1719, N 7, 2018.

[26] Кочаровский В.В., Железняков В.В., Кочаровская Е.Р., Кочаровский В.В. // УФН. 2017. Т. 187. С. 367-410; Kocharovsky V.V., Zheleznyakov V.V., Kocharovskaya E.R., Kocharovsky V.V. // Phys. Usp. 2017. V. 60. P. 345-384.

[27] Dicke R.H. // Phys. Rev. 1954. V. 93. P. 99.

[28] BlombergenN., Pound R.V. // Phys. Rev. 1954. V. 95. P. 8.

[29] Shishmarev D., Otting G.J. // Magn. Reson. 2011. V. 213. P. 76.

[30] Krishnan V., Murali N. // Prog. Nucl. Magn. Reson. Spectrosc. 2013. V. 68. P. 41.

[31] Ren L. et al. // Phys. Rev. B. 2013. V. 87. P. 161401.

[32] Leymann H.A.M., Foerster A., Jahnke F., Wiersig J., Gies C. Phys. Rev. Applied. 2015. V. 4. P. 044018.

[33] Rehler N.E., Eberly J.H. // Phys. Rev. A. 1971. V. 3. P. 1735.

[34] Bonifacio R., Lugiato L. // Phys. Rev. A. 1975. V. 11. P. 1507.

[35] Mac Gillivray J.C., Feld M.S. // Phys. Rev. A 1976. V. 14. P. 1169.

[36] Gross M., Haroche S. // Physics Reports. 1982. V. 93. P. 301.

[37] Андреев А.В., Емельянов В.И., Ильинский Ю.А. Кооперативные явления в оптике: Сверхизлучение. Бистабильность. Фазовые переходы. М.: Наука. Гл. ред. физ.- мат. лит., 1988); Andreev A.V., Emelyanov V.I., Ilinskii Yu.A. Collective Effects in Optics: Superradiance and Phase Transitions. Bristol: Institute of Physics Publishing, 1993.
[38] Benedict M.G., Ermolaev A.M., Malyshev V.A., Sokolov I.V., Trifonov E.D. Super-radiance Multiatomic Coherent Emission, CRC Press, 1996.

[39] Железняков В.В., Кочаровский В.В., Кочаровский В.В. // УФН. 1989. Т. 159. С. 193-260; Zheleznyakov V.V., Kocharovskii V.V., Kocharovskii V.V. // Sov. Phys. Usp. 1989. V. 32. P. $835-870$.

[40] Ariunbold G.O., Yang W., Sokolov A.V., Sautenkov V.A., Scully M.O. // Phys. Rev. A. 2012. V. 85. P. 023424.

[41] Архипов Р.М, Архипов М.В, Бабушкин И. Пахомов А.В., Розанов Н.Н. // Опт. Спектр. 2020. Т. 128. В. 4. С. 541-547; Arkhipov R.M., Arkhipov M.V., Babushkin I., Pakhomov A.V., Rosanov N.N. // Optics and Spectroscopy. 2020. V. 128. N 4. P. 529-535.

[42] Ginzburg V.L., Zheleznyakov V.V. // Comments Astrophys. Space Sci. 1970. V. 2. P. 197.

[43] Ginzburg V.L., Zheleznyakov V.V. Ann. Rev. Astron. Astrophys. 1975. V. 13. P. 511.

[44] Bloch F. // Phys. Rev. 1946. V. 70. P. 460.

[45] Eberly J.H. // Am. J. Phys. 1972. V. 40. P. 1374.

[46] Orszag M. J. Phys. A: Math. Gen. V. 1979. V. 12. P. 2205.

[47] Mak A., Shamuilov G., Sal'en P., Dunning D., Hebling J., Kida Y., Kinjo R., Mc Neil B.W., Tanaka T., Thompson N. et al. // Reports on Progress in Physics. 2019. V. 82. P. 025901.

[48] Dunning D.J., Mc Neil B.W.J., Thompson N.R. // Phys. Rev. Lett. 2013. V. 110. N 10. P. 104801.

[49] Бессонов Е.Г. // ЖЭТФ. 1981. Т. 80. № 3. С. 852-858. 1981; Bessonov E.G. // Sov. Phys. JETP. 1981. V. 53. N 3. P. 433-436.

[50] Архипов Р.М., Архипов М.В., Шимко А.А., Пахомов А.В., Розанов Н.Н. // Письма в ЖЭТФ. 2019. Т. 110. № 10. C. 9-20; Arkhipov R.M., Arkhipov M.V., Shimko A.A., Pakhomov A.V., Rosanov N.N. // JETP Lett. 2019. V. 110. N 1. P. $15-24$.

[51] Архипов Р.М, Архипов М.В, Розанов Н.Н. // Квант. Электрон. 2020, 50(9). С. 801-815; Arkhipov R.M., Arkhipov M.V; Rosanov N.N. // Quantum Electron. 2020, 50(9). P. 801-815.

[52] Chai X., Ropagnol X., Raeis-Zadeh S. M., Reid M., Safavi-Naeini S., Ozaki T. // Phys. Rev. Lett. 2018. V. 121. N 14. P. 143901.

[53] Розанов Н.Н. // Опт. Спектр. 2018. Т. 124. № 1. С. 75-77; Rosanov N.N. // Opt. Spectr. 2018. V. 124. N 1. P. 72-74.

[54] Arkhipov R.M., Arkhipov M.V., Babushkin I., Demircan A., Morgner U., Rosanov N.N. // Opt. Lett. 2019. V. 44. N 5. P. 1202.

[55] Розанов Н.Н., Высотина Н.В. // ЖЭТФ. 2020. Т. 157. N 1. C. 63-66; Rosanov N.N., Vysotina N.V. // JETP. 2020. V. 130. N-1. P. 52-55.

[56] Arkhipov R., Pakhomov A., Arkhipov M., Demircan A., Morgner U., Rosanov N., Babushkin, I. // Optics Express. 2020. V. 28. N 11. P. 17020-17034.

[57] Arkhipov R., Pakhomov A., Arkhipov M., Rosanov N. // Las. Phys. Lett. 2020, in press.

[58] Архипов Р.М., Архипов М.В., Розанов Н.Н. // Письма в ЖЭТФ. 2020. Т. 111. В. 9. C. 586-590; Arkhipov R.M., Arkhipov M.V., Rosanov N.N. // JETP. Lett. 2020. V. 111. N 9. P. 484-488.

[59] Ярив А. Квантовая электроника. М.: Сов. радио. 1980. Yariv A. Quantum electronics. Wiley, 1989.

[60] Розанов Н.Н. Диссипативные оптические солитоны от микро- к нано- и атто-. М.: Физматлит. 2011. 
[61] Manassah J.T. // Phys. Rev. A. 2014. V. 89. P. 053815.

[62] Manassah J.T. // Phys. Lett. A. 2014. V. 378. P. 2085.

[63] Manassah J.T. // Phys. Rev A. 2014. V. 90. P. 065801.

[64] Фриш С.Э. Оптические спектры атомов. М.-Л.: Государственное издательство физико-математической литературы, 1963.

[65] Собельман И.И. Введение в теорию атомных спектров. М.: Наука. 1977. 